\title{
Endoscopic removal of sharp metallic foreign bodies
}

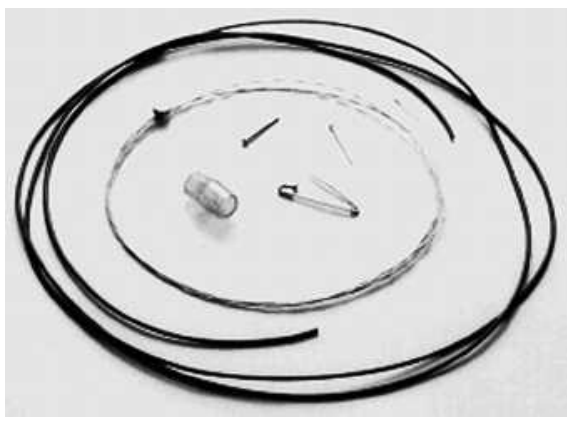

Figure 1 The ferromagnetic foreign bodyretrieval instrument, showing the Teflon sleeve, the ligator cylinder, and examples of the sharp foreign bodies removed.

Metallic foreign bodies have been removed under fluoroscopic control but this can be hazardous $[1,2]$. We describe here an endoscopic method for removal of sharp metallic foreign bodies using a magnetic retrieval instrument we designed ourselves.

After the trial was approved by the ethical committee and informed consent was obtained, ten patients who had ingested sharp foreign bodies were included in the study. Four people had ingested pins, four had ingested open safety pins, and two had ingested nails. Two were impacted and two were penetrating. Pentazocine $(15 \mathrm{mg})$ and hyosine butylbromide $(20 \mathrm{mg}$ ) were used as premedication. The time required, and the complication and failure rates were recorded.

We used a stainless steel wire $(200 \mathrm{~cm}$ in length, $0.75-\mathrm{mm}$ thick) with two 7-mmdiameter magnetic discs with a strength of 0.17 tesla fixed at one end. A Teflon sheath $(7 \mathrm{Fr}, 160 \mathrm{~cm}$ ) was used as a sleeve, and a ligator cylinder was used during foreign-body retrieval (Figure 1). The cylinder was fixed at the tip of the endoscope. The sheath was passed through the channel to the tip of the endoscope. The wire end of the instrument was passed through the sheath so that the magnet was positioned at the tip of the endoscope. As the endoscope reached the foreign body, the magnet was pushed out

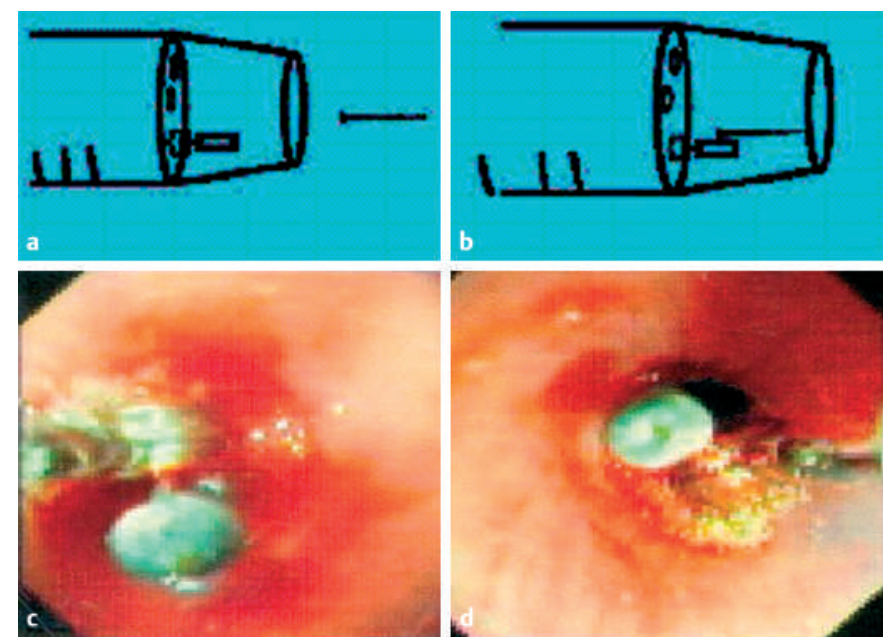

Figure 2 Diagramatic representation of the ferromagnetic foreign body-retrieval instrument, showing the retrieval of a pin $(\mathbf{a}, \mathbf{b})$. The endoscopic views show a pin (c) and a safety pin (d) during retrieval with the instrument.

and the foreign body stuck to the magnet. The foreign body and magnet were then drawn into the cylinder and the endoscope was removed. All the foreign bodies were removed using this method without any complications in mean time of $68 \mathrm{sec}-$ onds (range 46-100 seconds).

We used our instrument previously to remove ferromagnetic coins $[3,4]$. The sharp foreign bodies were light and so were attracted by the small magnet, and the pulling back of the magnet led to their disimpaction. Even the sharp foreign bodies that had penetrated could be pulled out because the head portion of the pins and safety pins could be visualized. The portion of the foreign body with a larger surface area had a tendency to stick to the magnet, meaning that the elongated sharp foreign bodies aligned themselves along their longitudinal axis (Figure 2). The sharp end of the foreign body followed the head, thus avoiding mucosal injury.

\section{Endoscopy_UCTN_Code_TTT_1AO_2AL}

\section{S. Nijhawan, V. Singh, P. Mallikaarjun, P. Jain, M. Tandon, M. Rastogi,}

\section{A. Mathur, R. R. Rai}

Department of Gastroenterology, SMS Hospital and Medical College, Jaipur, India.

\section{References}

${ }^{1}$ Paulson EK, Jaffe RB. Metallic foreign bodies in the stomach: fluoroscopic removal with a magnetic orogastric tube. Radiology 1990; 174: $191-194$

2 Volle E, Hanel D, Beyer P, Kaufmann HJ. Ingested foreign bodies: removal by magnet. Radiology 1986; 160: 407-409

${ }^{3}$ Nijhawan S, Joshi A, Shende A et al. Endoscopy-assisted ferromagnetic foreign body removal with novel magnetic instrument. Endoscopy 2004; 36: 1130

${ }^{4}$ Nijhawan S, Rastogi M, Tandon M et al. Magnetic loop basket: a "two-in-one" instrument. Endoscopy 2006; 38: 723-725

\section{Corresponding author}

\section{S. Nijhawan}

Department of Gastroenterology, SMS Hospital and Medical College

112 Panchsheel Enclave

Gokul Bhai Bhatt Marg

Durgapura

Jaipur

India

Fax: $\quad+91-0141-2561024$

Email: dr_nijhawan@yahoo.com 